\title{
Internet resources for the space sciences
}

\author{
By Robert W. Pasicznyuk
}

\section{A guide to information that is out of this world}

I $\mathrm{n}$ the coming decade NASA expects to download a terabyte of information each day from its space station-roughly equivalent to 71 billion floppy disks or 15 billion compact discs. The deluge of tomorrow has a harbinger today in the wealth of information opportunities in the space sciences on the Internet. Internet aerospace and astrophysics sources offer a mix of traditional text databases, high-resolution graphics archives, news services, and discussion lists. Like other information in electronic formats, space sciences sources on the Net have the advantage of being continually updated and augmented. The varied formats present media ready for personal manipulation, analysis, and enjoyment.

Information sources on the Internet in the space sciences offer an array of text, auditory, and graphic information. Taking advantage of these sources requires both the computer hardware and software to handle multimedia files. One of the givens of multimedia computing is that the files are large. Searchers interested in collecting multiple files must have sufficient storage capacity to handle the load.

\section{Multimedia information centers}

CASS: Center for Advanced Space Studies. Cass offers text and graphic information from numerous NASA projects. Contributors include the Lunar and Planetary Institute (LPI), the Exploration Science Institute (ESI), NASA's Division of Educational Program (DEP), and NASA libraries. Through CASS, users can find an- nouncements of astrophysics and astronomy professional meetings, read articles from the Lunar and Planetary Bulletin, or consult several indexes and reference works. CASS also acts as a gateway to other sources: the Lunar and Planetary Bibliography, NASA libraries, and the Image Retrieval and Processing System. ACcess: Telnet: CASS.JSC.NASA.GOV; login as cass (lowercase); the password is online. (Note: The "About CASS" option is invaluable for navigating the system.)

NASA SPACELINK. Spacelink provides a wealth of text reports covering NASA projects both past and present. In addition to astronomy and astrophysics information, users can display files covering the environment, the history of space exploration, space shuttle flight information, NASA plans for the future, and fact sheets. Spacelink also provides image files (GIF format). Look for images of the comet ShoemakerLevy 9's impact with Jupiter. Access: Telnet: SPACELINK.MSFC.NASA.GOV; anonymous ftp is available at spacelink.msfc.nasa.gov; connect via the World Wide Web at http://spacelink. msfc.nasa.gov.

NED: NASA Extragalactic Database. NED allows users to search celestial objects, data, and literature. Information about objects is accessible by object name, position, and skyplot. Search parameters also include catalog and photometry. NED acts as a current-awareness service providing contents and abstracts information from prominent astronomy and astrophysics journals. Searching the journal contents, abstracts, and dissertation/thesis options will become apparent to anyone who has searched similar clatabases. The other options require facility with astronomy nomenclature and designations. More information about NED is avail- 
able in Egret and Albrecht's Databases and Online Data in Astronomy. Page 91 contains a discussion of NED. Access: Telnet: NED.IPAC. CALTECH.EDU; login as ned

NODIS: National Space Science Data Center. NODIS is a gateway for space science data in the topics of astrophysics, space physics, planetary sciences, earth sciences, microgravity, and spacecraft. NODIS also provides a catalog of NASA CD-ROMs with ordering capability. Users can view sample images of the CDs through Mosaic. Otherwise, the images can be downloaded and retrieved via anonymous ftp. NODIS files are in GIF, Postscript, RTF, and ASCII formats. Access: Telnet: NSSDCA.GSFC.NASA.GOV; login as nodis. Anonymous $\mathrm{ftp}$ is available at nssdc.gsfc. nasa.gov.

STEIS: Space Telescope Electronic Information Service Gopher. The Space Telescope Information System gopher is maintained by the Space Telescope Science Institute (STSI). It contains information about and from the Hubble Space Telescope. Through the gopher, users can more easily locate, view, and download files. They can search Hubble status reports, time lines, and action reports. The STEIS gopher also provides an index to some astrophysics and astronomy journals, an astronomer e-mail address index, and a gateway to other astronomy databases and fip archives. Some of the unique sources available via the STEIS gopher are electronic PictureBooks. Produced by the Special Studies Office of the STSI, the PictureBooks offer Hypercard tours of a variety of space and earth sciences subjects. Look for Hubble Space Telescope images, views of the earth from the space shuttle Endeavour, images of planets, and more. Access: Gopher: stsci.edu; anonymous ftp is also available at stsci.edu; STEIS is accessible via the World Wide Web at gopher://stsci.edu:70/1.

ADS: NASA Astrophysics Data System Server. The NASA ADS aims at making NASA space missions information available to astronomers. It supplies software allowing users to make complex operations and manipulations on NASA data sets.

Sample questions that can be answered through ADS include: What supernova remnant images are available in the $\mathrm{X}$-ray wavelength band? What sites have catalogs of observational data? Are the observations available?

ADS also provides access to its home page, tutorial, and registration. The home page sup- plies information about the system and acts as a gateway to other Internet space sciences sources. ADS also allows searching of 160,000 abstracts produced in the NASA/STI project, access to an archive of data collected by the Einstein X-ray satellite mission, and access to about 150 astronomical catalogs. The home page describes how to register for ADS software. Access: http://adswww.colorado.edu.

\section{News and discussion}

Hubble Telescope Bulletin Board Daily and Status Reports. This BBS lists Hubble status reports, scheduled activities, and tasks accomplished. Access: Telnet: STINFO.HQ.ESO.ORG; login as stinfo (lower case)

NASA SPACELINK. SPACELINK provides NASA news and shuttle flight information. $A C$ cess: Telnet: SPACELINK.MSFC.NASA.GOV.

SPACEMET: Science and Space Bulletin Board. SpaceMet is sponsored by the Department of Physics and Astronomy at the University of Massachusetts, Amherst, the National Science Foundation, and MassNet. It provides a forum for discussing space sciences issues. This BBS is geared toward $\mathrm{K}-12$ students and teach ers, but many of the discussions are appropri ate for students and faculty at the collegiate level who have interest in the subject. Access: Telnet: SPACEMET.PHAST.UMASS.EDU.

SCI.ASTRO.HUBBLE Newsgroup This forum was originally intended to center on the processing of data taken with the Hubble Space Telescope (HST). It has become an instrument for the dissemination of information about the operations and status of HST. The newsgroup also serves as a platform for problems, requests, suggestions, and needs of the scientific community as it uses the telescope for research purposes. Contact: scowen@wfpc3.la.asu.edu for information and article submission.

SpaceNews. Not a listserv or BBS, SpaceNews is a news and information service available weekly via the Amateur Packet Radio Network (AMPR), AMSAT-OSCAR-16, and the Usenet newsgroups of rec.radio.amateur.space, rec.radio.amateur.misc, rec.radio.amateur.info, and sci.space.news. Archives are available via anonymous ftp at pilot.njin.net in the pub/ SpaceNews subdirectory. It originates at KD2BD in Wall Township, New Jersey, and is made available for unlimited free distribution. Access: Finger magliaco@pilot.mjin.net for current news. Contact: kd2bd@ka2qhd.de.com or kd2bd@amsat.org for more information. 


\title{
MANY OF AMERICA'S YOUTH \\ ORGANIZATIONS TEACH LOYALTY.
}

\author{
BRAVERY.
}

\section{AND SURVIVAL.}

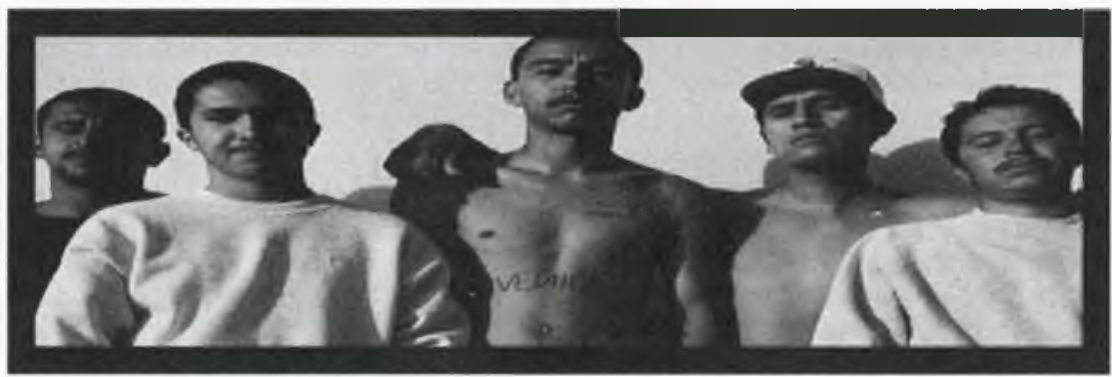

BUt IN SOME, THE DUES ARE HIGHER THAN OTHERS.

Twenty years ago, the closest most kids came to a gang was hanging out with friends in a clubhouse. Today, tens of thousands are gang members, with more joining every day.

The growth of gangs across America is just one of the important public and social policy issues you can research through PAIS International. PAIS gives you immediate access to an index of some 400,000 hard-to-find articles,

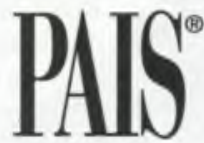

books, government documents, statistical directories and more. It references literature published around the globe. And now it's even available on leased tape.

So if you're looking for information, stay out of trouble. Use PAIS.

Public Affairs Information Service, Inc. 521 West 43rd Street, New York, NY 10036-4396 800-288-PAIS, 212-736-6629 (in NYC)

FAX: 212-643-2848, e-mail: inquiries@pais.org

\section{NO ONE LOOKS AT THE WORLD LIKE PAIS}

